\section{Ante la prevista erradicación de la poliomielitis}

La Organización Mundial de la Salud, en colaboración con los Centros para el Control y la Prevención de Enfermedades de los Estados Unidos de América, el club Rotary International y gobiernos de diversos países del mundo, está en las etapas finales del proyecto de erradicación de la poliomielitis iniciado en 1988. Si se mantienen los niveles actuales de recursos asignados y de cooperación continua, en el ya próximo 2003 ese objetivo se habrá alcanzado. En todo el mundo se registraron solo 4000 casos de poliomielitis en 1996, lo que significa una disminución de casi $90 \%$ con respecto a los 35000 casos notificados en 1988. La erradicación de la poliomielitis en la Región de las Américas fue certificada en 1994 y en la actualidad la circulación del virus salvaje está limitada al sur de Asia y a África. Como la cesación ulterior de medidas de control es inherente al concepto de erradicación, la posibilidad de suspender la vacunación ya ha sido planteada en el curso de reuniones de expertos llevadas a cabo en 1996 y 1997. Si así se procediera, desde el año 2003 en adelante quedaría disponible una infraestructura médica que se podría utilizar para una campaña contra el sarampión. Las existencias de poliovirus en laboratorios se destruirían, o bien su almacenamiento quedaría restringido a condiciones de extrema seguridad.

Dove y Racaniello expresan sus reservas acerca de esas perspectivas en un artículo publicado en el mismo número de Science (1997;277: 779-780), al cual se refieren los presentes comentarios de Hull y Aylward. El hecho de que la campaña de erradicación se fundamenta en el uso exclusivo de la vacuna oral de Sabin a base de virus vivos hace imposible eliminar totalmente la circulación de cepas vacunales, y es reconocido que pueden mutar hacia la virulencia. En los países desarrollados, estas son la única causa de poliomielitis (1 caso por 300000 a 500000 vacunados). Para Dove y Racaniello, el concepto de erradicación debería estar asociado no solo con las cepas salvajes sino también con las vacunales. Como ese objetivo es imposible de lograr con la vacunación oral (se eligió por su menor costo y por no requerir personal adiestrado ni agujas estériles), ellos proponen que se continúe hasta tanto pueda asegurarse la vacunación con cepas inactivadas (Salk). También manifiestan sus dudas acerca de lograr una completa eli- minación de las existencias de poliovirus en laboratorios y esa es condición imprescindible para prevenir reintroducciones eventuales del virus en un mundo que habría dejado de estar protegido.

Con respecto a esas objeciones, Hull y Aylward responden que, precisamente ante la posibilidad de que las existencias de poliovirus en los laboratorios del mundo deban ser eliminadas, la OMS ha iniciado una consulta con productores de vacuna, organizaciones técnicas e institutos de investigación. En cuanto a la persistencia de las cepas Sabin, si bien algunos vacunados inmunocomprometidos las han excretado por 2 años o más, no parecería que puedan circular por mucho tiempo en una población general, ni tampoco causar brotes de enfermedad paralítica. En campañas masivas de vacunación en Cuba y en Hungría, solo en los dos o tres meses siguientes pudieron ser aisladas esas cepas de muestras humanas y ambientales. Con respecto al reemplazo de la vacuna Sabin por la Salk, no es una decisión que pueda adoptarse sin una rigurosa evaluación previa, ya que la capacidad de producción de vacuna inactivada es bastante limitada y tendría que ser ampliada para poder disponer de cantidad suficiente para todo el mundo. De todas formas y dada la importancia del riesgo teórico que supone el cese de la práctica de inmunización, la OMS está patrocinando estudios para determinar cómo y cuándo proceder de forma adecuada. Se están investigando, por ejemplo, la prevalencia y duración de la excreción de virus vacunales por personas inmunodeficientes tanto en países industrializados como en desarrollo. Se está estimando también el intervalo entre la administración de la vacuna y la recuperación del virus en heces, en casos de niños con enfermedad paralítica por virus vacunal.

Como han dicho Dove y Racaniello, "El control de la poliomielitis ha mejorado sustancialmente la calidad de vida en todo el mundo. Una vez concluida esa tarea, los países en desarrollo podrán orientar sus esfuerzos hacia otros problemas de salud pública. Sin embargo, para que la erradicación de la poliomielitis sea realmente exitosa, tiene que lograrse en el contexto equilibrado de una campaña más amplia para mejorar la salud y las condiciones sanitarias de la población. (Hull HF, Aylward RB. Ending polio immunization. Science 1997; 277:780; Dove AW, Racaniello VR. The polio eradication effort: should vaccine eradication be next? Science 1997; 277:779-780.) 


\section{Vacunación BCG y enfermedades atópicas}

Las tasas de enfermedad atópica mediada por anticuerpos IgE están aumentando en los países desarrollados y la proporción de niños con asma, dermatitis atópica, rinoconjuntivitis y alergia alimentaria ya excede de $30 \%$. Ese incremento, cuya causa se ignora, podría en parte deberse a la disminución de ciertas enfermedades infecciosas y también a cambios en los planes de inmunización (entre ellos, la vacunación antituberculosa con BCG). Por ser un potente inductor de la inmunidad mediada por células en general y del interferón- $\gamma$ en particular, la BCG también ha sido empleada para tratar de modular la respuesta inmunitaria y, sobre esa base, interferir en la patogenia de determinadas enfermedades. A ese respecto, se ha logrado que una dosis subcutánea única de BCG produzca una remisión clínica a largo plazo en niños con diagnóstico reciente de diabetes tipo 1. En Suecia, la BCG llegó a aplicarse a 95\% de los recién nacidos, hasta que en 1975 se interrumpió esa práctica por los efectos colaterales de la vacuna y, sobre todo, por el bajo riesgo de exposición a la tuberculosis. En la actualidad, la tasa de vacunación con BCG es menor de $4 \%$, ya que solo se administra a niños en estrecho contacto con tuberculosos o a inmigrantes de zonas de alto riesgo, así como en el caso de que lo soliciten los padres.

Teniendo en cuenta esas condiciones, Alm et al. emprendieron una investigación para establecer si la vacuna BCG administrada en la infancia más allá del período neonatal era un estímulo suficiente para desviar al sistema inmunitario de niños con alto riesgo hacia un fenotipo no atópico. Para ello, estudiaron retrospectivamente una cohorte de 216 niños con antecedentes hereditarios de atopia, nacidos en Estocolmo entre 1989 y 1992. Estos niños habían recibido BCG antes de los 6 meses de edad. El grupo control, 358 niños apareados por edad con los casos, no había sido vacunado. Todos los niños habían acudido a consulta hospitalaria durante el período de 1995 a 1996 para la evaluación de sus antecedentes y signos clínicos de enfermedad atópica.

La vacunación temprana no afectó al desarrollo de la enfermedad atópica en niños preescolares con antecedentes atópicos, según se estableció por examen clínico, pruebas cutáneas y análisis de anticuerpos IgE. Estos resultados difieren de los encontrados por Shirakawa et al. (Science 1997;275:77-79) en escolares japoneses de 12 a 13 años, en quienes se observó una relación inversa entre la reacción de Mantoux positiva y varios componentes de atopia, entre ellos IgE específica y síntomas. Para Alm et al., la discrepancia sería atribuible a que la incidencia de tuberculosis en el Japón es alrededor de 10 veces mayor que en Suecia, lo que significaría una mayor exposición natural a la micobacteria. La diferencia de efectos consecutivos a la vacunación y a la infección natural ha sido bien ilustrada por un estudio retrospectivo de niños de Guinea-Bissau (Lancet 1996;347:1792-1796), donde la infección por sarampión, pero no la vacunación antisarampionosa, estuvo asociada con reducción de la atopia. Los hallazgos negativos de Alm et al. corresponden a niños en edad preescolar. Si los resultados fueran similares entre niños mayores y niños sin atopia hereditaria, podría concluirse que la vacunación BCG no constituye una estrategia primaria efectiva para prevenir la atopia.

Sin embargo, Silverman (Lancet 1997;350:381382), a la vez que reconoce la calidad del estudio estadístico efectuado por Alm et al., opina que debe ser ampliado para incluir casos con bajo riesgo de atopia, ya que una elevada proporción de niños asmáticos y la mayoría de los niños atópicos provienen de familias sin antecedentes hereditarios. Podría ocurrir que esas poblaciones infantiles fueran más susceptibles a la modulación de la respuesta inmunitaria, ya sea por manipulación ambiental o por efecto de agentes terapéuticos. Aunque se requieren pruebas en modelos no humanos y también in vitro para redefinir las relaciones entre factores del huésped (genotipo y condición inmunitaria), estímulos ambientales (dieta y adyuvantes respirados o ingeridos), y factores infectantes o vacunales (carga, oportunidad y pureza), será muy útil el aporte representado por datos que se obtengan de poblaciones de diversos países, dados los diferentes programas de vacunación BCG instituidos e incluso su restricción a recién nacidos con alto riesgo de tuberculosis. (Alm JS, Lilja G, Pershagen G, Scheynius A. Early BCG vaccination and development of atopy. Lancet 1997;350:400-403.)

\section{Cambios demográficos y epidemiológicos en América Latina}

Las variaciones demográficas y socioeconómicas contribuyen a largo plazo a cambiar los patrones de salud y enfermedad, y dan lugar a la situación que se ha denominado transición epidemiológica. En general, la disminución de la mortalidad por enfermedades infecciosas, especialmente en los niños, ha mejorado la esperanza de vida. En la mayoría de los países de América Latina asumen cada vez más importancia las enfermedades crónicas y degenerativas. La transición epidemiológica, caracterizada por la coexistencia de enfermedades crónicas y accidentes con enfermedades infecciosas, varía en función del ritmo de los cambios demográficos y de los 
procesos de urbanización e industrialización. Por eso, Albala et al. diseñaron un estudio descriptivo poblacional para comparar la situación transicional de Chile, Guatemala, México y Uruguay (países en distintas etapas de transición epidemiológica) con los Estados Unidos de América y Canadá (países desarrollados en etapa epidemiológica avanzada). Como fuente de datos demográficos, socioeconómicos y de mortalidad, los autores recurrieron a información oficial de organizaciones nacionales e internacionales, tales como el Banco Mundial, la OPS, las Naciones Unidas, el Centro Latinoamericano de Demografía, el Instituto Nacional de Estadística y otros registros gubernamentales.

De acuerdo con los resultados obtenidos, Guatemala se encuentra en una etapa pretransición con una elevada proporción de enfermedades transmisibles entre las causas de muerte $(61 \%)$, en relación con México (22\%), Chile (13\%) y Uruguay $(7 \%)$. México se ubica en una situación de prolongada transición, en tanto que Chile se aproxima a Uruguay en su acceso al período postransición. Pese a las tasas decrecientes de mortalidad, la proporción de muertes por enfermedades crónicas y accidentes ha ascendido a 30\%, excepto en Uruguay. En lo que se refiere a la mortalidad por enfermedades cardiovasculares y cáncer, en Chile y Uruguay las tasas ajustadas por edad son similares a las registradas en los Estados Unidos y Canadá, pero mayores que en México y Guatemala.

En los cambios de los patrones de salud influye primero la transición demográfica, cuando disminuyen la mortalidad por enfermedades infecciosas y la fecundidad, y, en segundo lugar, la epidemiológica, cuando las enfermedades crónicas empiezan a predominar como causas de muerte. Los patrones de salud de los países de América Latina están profundamente influidos por ambas transiciones. En muchos países, el descenso de las tasas de mortalidad ha precedido al de la natalidad, en ocasiones por dos o más decenios, con el consiguiente aumento de la población ( $3 \%$ o más al año). A medida que disminuye la fecundidad aumenta la población mayor de 65 años y con ello la carga de enfermedades no transmisibles. Según los autores, en América Latina no se ha otorgado suficiente importancia a las intervenciones que podrían prevenir la alta prevalencia de factores de riesgo de enfermedades crónicas. Para las próximas décadas es de esperar un incremento de las enfermedades no transmisibles. Por lo tanto, se necesita analizar profundamente la transición demográfica y epidemiológica, con el fin de adecuar las políticas de salud a las nuevas situaciones de pretransición, transición y postransición. (Albala C, Vio F, Yáñez M. Transición epidemiológica en América Latina: comparación de cuatro países. Rev Med Chile 1997;125:719-727.)

\section{Valor predictivo del metanálisis}

Los grandes ensayos aleatorizados controlados se consideran habitualmente como regla de oro para evaluar la eficacia de intervenciones clínicas. Sin embargo, como las exigencias de ese tipo de ensayo no son fáciles de cumplir, el metanálisis ha recibido una amplia aceptación por parte de estadísticos y clínicos, ya que es un método desarrollado de forma que permite resumir los resultados de diferentes estudios sobre un tema de interés. Los críticos alegan que los resultados combinados incorporan los sesgos de los estudios individuales incluidos y se convierten en nuevas fuentes de sesgo, lo que se debe principalmente a la selección de estudios inevitablemente heterogéneos.

Aunque mucho se ha comentado a propósito de las ventajas y desventajas de los metanálisis, pocos estudios han comparado sistemáticamente los resultados de metanálisis de varios ensayos pequeños con los resultados de grandes ensayos aleatorizados controlados. En consecuencia, LeLorier et al. emprendieron un estudio para comparar los resultados de una serie de esos grandes ensayos aleatorizados (de 1000 pacientes o más) con los resultados de metanálisis correspondientes ya publicados. Limitándose a cuatro revistas importantes (New England Journal of Medicine, Lancet, Annals of Internal Medicine y Journal of the American Medical Association) y al período de 1991 a 1994, se encontraron 12 informes de ensayos y 19 metanálisis sobre temas similares, que coincidían en cuanto a poblaciones estudiadas, intervenciones terapéuticas y por lo menos un resultado.

De acuerdo con el análisis efectuado por LeLorier et al., si no se hubieran realizado posteriores ensayos aleatorios y controlados, los metanálisis habrían llevado a adoptar tratamientos ineficaces en $32 \%$ de los casos $(100 \%$ menos el valor predictivo positivo), y al rechazo de uno útil en 33\% (100\% menos el valor predictivo negativo). Es importante reconocer que estas medidas de divergencia, por haber tenido en cuenta la perspectiva propia de una decisión médica ulterior, tienden a sobrestimar el grado de discrepancia estadística, ya que en ningún caso se dieron divergencias tales que llegaran a implicar respuestas opuestas estadísticamente significativas, según se tratara de ensayos aleatorios o metanálisis. Sin embargo, los resultados del estudio parecen indicar que un resumen de toda la información contenida en un conjunto de ensayos puede tender hacia una simplificación excesiva y por ello al riesgo de una conclusión inapropiada. De ahí se 
desprende que debe considerarse con cierta cautela la información aportada por un metanálisis. Para Cook et al. (Chest 1995;108:suppl:227S-230S), corresponde estimarla en función de la consistencia de los resultados de los estudios incluidos, mientras que Horwitz (Am J Med 1987;82:498-510) piensa que cada ensayo debe evaluarse por separado. Si bien estas son tareas indudablemente laboriosas, es evidente que permitirían discriminar de forma válida los efectos de un medicamento y con ello que los resultados de la investigación fueran realmente útiles para el médico clínico en la práctica diaria. (LeLorier J, Grégoire G, Benhaddad A, Lapierre J, Derderian F. Discrepancies between meta-analyses and subsequent large randomized, controlled trials. N Engl J Med 1997; 337:536-542).

\section{El sida en la comunidad latina de los Estados Unidos de América}

En diciembre de 1996, de 581429 casos de sida notificados a los Centros para el Control y la Prevención de Enfermedades de los Estados Unidos, 103023 provenían de la población latina. Aunque esta solo representa $10 \%$ de los habitantes del país, en la comunidad latina se registran $18 \%$ de los casos de sida, con un pronóstico de enfermedad más grave que para todos los demás grupos étnicos, exceptuados los afroamericanos. Es probable que la prevalencia real de la enfermedad sea aun mayor, ya que muchos latinos no tienen acceso a las pruebas de detección de VIH ni a cuidados sanitarios apropiados (MMWR 1997;46:165-173). En 1996, de los 12966 adolescentes y adultos latinos diagnosticados de sida, 31,8\% habían nacido en los Estados Unidos, $28,5 \%$ en Puerto Rico, 8,5\% en México, 2,9\% en Cuba y $6,2 \%$ en Centroamérica o América del Sur.

La vía principal de transmisión del VIH en varones latinos son las relaciones sexuales con otros hombres $\mathrm{y}$, en segundo término, el consumo de drogas por vía parenteral. Los puertorriqueños son la excepción, ya que la transmisión del VIH debida al consumo de drogas por vía intravenosa es tres veces más frecuente que la atribuible a relaciones homosexuales. Para las mujeres latinas, el mayor factor de riesgo es el uso endovenoso de drogas, seguido en orden de importancia por relaciones sexuales con un consumidor actual o antiguo de drogas por vía parenteral y las relaciones sexuales con un hombre que no consume drogas por esa vía.

De acuerdo con la revisión de la bibliografía que han realizado Sánchez y Pérez, entre los factores determinantes de la mayor incidencia de sida en la población latina se cuentan un menor nivel de conocimientos generales sobre la enfermedad y su forma de transmisión, una mayor tasa de infecciones de transmisión sexual y la falta de adecuación de los programas preventivos a las necesidades culturales del grupo. Si bien los profesionales de la salud gozan de una alta credibilidad en la comunidad, los médicos latinos son en general renuentes a evaluar sus pacientes en función del riesgo de infección. Sería deseable que adoptaran un papel más activo, ya que probablemente son los responsables de atender a $80 \%$ de los latinos en los Estados Unidos.

Luego de una evaluación de los factores que favorecen el riesgo de contraer la infección y de empeorar el pronóstico de la enfermedad, los autores concluyen que los factores socioeconómicos, lingüísticos y culturales deben ser tenidos en cuenta para toda intervención dirigida a la prevención, el diagnóstico y el tratamiento del sida en ese grupo étnico. (Sánchez MR, Pérez G. Estado actual de la infección por el VIH en la población latina. MEDICO Interamericano 1997; 16:437-444.)

\section{Diagnóstico de encefalitis herpética por reacción en cadena de la polimerasa}

La introducción de la reacción en cadena de la polimerasa (RCP) como prueba para establecer el diagnóstico de encefalitis herpética ha permitido prescindir de la biopsia cerebral, un procedimiento invasor que solía ser el único medio de diagnóstico seguro de esa infección. Por otra parte, la detección de ácido desoxirribonucleico (ADN) viral por RCP en líquido cefalorraquídeo es más específica y sensible que otros métodos, incluido el aislamiento del virus de tejidos cerebrales obtenidos por biopsia. Este síndrome, que se caracteriza por comienzo súbito, fiebre, cefalea, convulsiones, signos neurológicos focales y alteración de la conciencia, había sido definido en función de los pacientes en quienes una biopsia muestra necrosis del lóbulo temporal y de la porción orbitaria del frontal. Es muy posible que estudios prospectivos basados en el diagnóstico por RCP permitan ampliar la gama reconocible de las manifestaciones clínicas de la encefalitis por virus del herpes simple.

Fue con ese fin que Domingues et al. analizaron los datos clínicos y neurodiagnósticos de 49 pacientes ingresados en ocho diferentes hospitales de São Paulo y Campinas, Brasil, por tener encefalitis focal (29), moderada (12) o difusa (8). La RCP del líquido cefalorraquídeo fue positiva en $51,7 \%$ de los pacientes con encefalitis focal y en $25 \%$ de aquellos con formas moderadas. El hecho de que el diagnóstico fuera confirmado en solo $36 \%$ de los casos puede que se haya debido a una tendencia médica a instituir el tratamiento con aciclovir en pacientes 
con formas moderadas y no focalizadas de encefalitis, actitud en parte explicable por el afán de iniciar el tratamiento lo más pronto posible, aun cuando no se contara con pruebas específicas para corroborar el papel causal del virus herpes simple. Pese a ello, la RCP permitió establecer ese diagnóstico en tres casos que, por su carácter moderado, no habían sido identificados por examen clínico. El análisis de los datos clínicos no mostró diferencias históricas o neurológicas entre los casos positivos por RCP y los negativos. En cuanto a las pruebas neurodiagnósticas, su correlación con RCP positiva fue de $75 \%$ para la electroencefalografía y de $88,9 \%$ para la imagen por resonancia nuclear.

Los autores concluyen que las muestras de líquido cefalorraquídeo de todos los pacientes que posiblemente tengan encefalitis herpética deben ser analizadas por RCP, ya que los datos clínicos y neurodiagnósticos no son capaces de establecer el diagnóstico de forma inequívoca. Una prueba como la RCP (no invasora, rápida, y de sensibilidad y especificidad elevadas) amplía los recursos diagnósticos del médico y justifica la pronta institución del tratamiento adecuado para la encefalitis por virus del herpes simple, lo cual asegura un mejor pronóstico para el paciente. (Domingues RB, Tsanaclis AMC, Pannuti CS, Mayo MS, Lakeman FD. Evaluation of the range of clinical presentations of herpes simplex encephalitis by using polymerase chain reaction assay of cerebrospinal fluid samples. Clin Infect Dis 1997;25:86-91.)

\section{¿Implica riesgo de adicción el tratamiento del dolor con opioides?}

El tratamiento del dolor y la medicina de la adicción constituyen áreas nuevas y dinámicas que comparten la preocupación por la respuesta humana a los opioides y otros medicamentos que pueden causar adicción. Sin embargo, los dos campos han evolucionado como disciplinas separadas y ello ha afectado negativamente a pacientes, clínicos, investigadores y a la sociedad en su conjunto. Los efectos adversos de la falta de comunicación entre las dos disciplinas imponen la necesidad de un cambio y esto fue discutido en la conferencia organizada por el recientemente creado Grupo de Trabajo sobre el Manejo del Dolor y la Dependencia Química, que tuvo lugar del 21 al 23 de noviembre de 1996 en la ciudad de Nueva York. Algunos de los integrantes de ese grupo son los autores de un oportuno análisis sobre la naturaleza del problema y las posibilidades de resolverlo, que aquí reseñamos.

El conocimiento acerca de las propiedades analgésicas de los opioides no implica un conocimiento simultáneo acerca de la vulnerabilidad a su adicción ni de las formas de empleo que puedan resultar aberrantes. De igual modo, la familiaridad con la adicción y la experiencia con la administración continua de opioides no siempre se acompaña del reconocimiento de normas ampliamente aceptadas para el uso de opioides en el manejo del dolor agudo o crónico. Esa falta de interacción entre las dos disciplinas puede llevar a leyes y acciones tendentes a reducir el abuso de su consumo, pero que en último término llegan a afectar la legítima necesidad de opioides en casos de dolor intratable. Aunque su empleo en pacientes de cáncer está apoyado por importantes organizaciones nacionales e internacionales, hasta $40 \%$ de esos pacientes no reciben opioides para el dolor y ese riesgo aumenta a $80 \%$ en los casos de sida con antecedentes de drogadicción. La ignorancia y el estigma comprometen el manejo del dolor agudo y crónico en pacientes que reciben una terapia de mantenimiento con metadona, aun cuando ellos también podrían responder favorablemente al tratamiento con opioides. Si bien la mayoría de los especialistas favorece el empleo a largo plazo de opioides en algunos pacientes con dolor crónico pero sin cáncer, esa postura sigue siendo motivo de una controversia que no se resolverá sin un conocimiento actualizado de los riesgos de adicción.

Ahora es el momento de establecer el diálogo necesario entre los especialistas en el manejo del dolor y los de la medicina de la adicción. El rápido avance de las neurociencias está desentrañando los mecanismos cerebrales que subyacen la vulnerabilidad a la adicción y la nocicepción. Se está creando así la oportunidad de establecer vínculos entre ambas disciplinas y a partir de ello de estimular nuevas investigaciones que fundamenten la base científica de la práctica clínica. Los programas educativos son esenciales para que ambos tipos de especialistas puedan juzgar la seguridad, eficacia y factibilidad de tratamientos analgésicos y también para los encargados de su legislación en la comunidad. Por medio de una comunicación más fluida, los clínicos podrán cumplir las regulaciones sin por ello sacrificar el alivio de sus pacientes, y los responsables de imponer alguna penalización eventual podrán controlar la práctica médica y reducir el consumo ilegal de drogas sin que sus acciones engendren temores en médicos y pacientes.

Además de la segunda conferencia anual que el Grupo de Trabajo en el Manejo del Dolor y la Dependencia Química proyecta para enero de 1998, otras actividades similares deberían ser puestas en marcha ya para posibilitar un menor manejo del dolor agudo y crónico en pacientes con y sin antecedentes de dependencia química. (Portenoy RK, Dole V, Joseph H, Lowinson J, Rice C, Segal S, et al. 
Pain management and chemical dependency. JAMA 1997;278:592-593.)

\section{La estabilidad de los medicamentos en climas tropicales}

Solamente $16 \%$ de los productos terapéuticos disponibles en los países en desarrollo están en la lista de los medicamentos esenciales, pero la calidad de estos fármacos es uno de los componentes más importantes de las políticas de salud de esos países. Si bien la calidad está asegurada por las compañías farmacéuticas cuando los fármacos arriban al país de destino, es posible que su almacenamiento, a veces por años en climas tropicales, ocasione pérdidas cuantitativas de sus ingredientes activos.

En un estudio reciente, Ballereau et al. procuraron verificar la estabilidad de 27 medicamentos esenciales en el clima tropical de la parte occidental de Burkina Faso. Durante un período de 2 años, los medicamentos fueron almacenados en dos farmacias, una en el hospital de un centro urbano y la otra en una clínica comunitaria rural. La elección de los dos lugares se basó en la necesidad de efectuar la evaluación según las condiciones locales prevalecientes y de comparar las diferencias entre el área urbana y la rural. Ambos depósitos estaban construidos con ladrillo y ninguno tenía aire acondicionado, pero el del hospital tenía una ventana y techo de madera laminada, mientras que el de la clínica no tenía fuente de luz externa y el techo era de metal. Los productos, que se almacenaron en sus empaques originales según se recibieron, se seleccionaron de la sexta lista de la OMS de medicamentos esenciales. Todos los días se registraban la temperatura y la humedad, y cada 3 meses se tomaban muestras de cada medicamento para su análisis en el Centro Colaborativo de la OMS para el Estudio de la Estabilidad de Medicamentos, de Nantes, Francia.

La temperatura fue mayor $\left(32-40^{\circ} \mathrm{C}\right)$ en la clínica rural, pero la humedad menor que en el hospi- tal, diferencias probablemente debidas tanto a condiciones del clima local como a la estructura de las instalaciones. El análisis cuantitativo mostró que en la mayoría de los medicamentos no hubo pérdida significativa de su ingrediente activo, pero sí se comprobó una pérdida cuantitativa mayor de 10\% en ampicilina, eritromicina, sulfaguanidina, furosemida inyectable, penicilina $G$, trimetoprima y cloroquina. Los medicamentos almacenados en la clínica rural estuvieron sometidos a temperaturas más altas y resultaron menos estables que en el hospital, pero la pérdida de ingrediente activo no fue mayor de $30 \%$ en ningún caso. De todos modos, las reducciones de ingredientes activos ocurrieron después de 270 días con excepción de la penicilina G y la trimetoprima, en los que se midieron pérdidas a los 90 días. La rápida degradación de estos dos medicamentos indica que requieren cuidado especial al almacenarlos.

Con respecto a los siete medicamentos en los que se comprobó pérdida de estabilidad y actividad, los autores concluyen que no conviene almacenarlos por más de un año. Sería aconsejable que en la práctica su entrega se hiciera dos veces al año y no una, como ocurre hasta ahora en el mejor de los casos. El estudio ha permitido identificar aquellas drogas que requieren monitoreo en climas tropicales para reducir el riesgo de pérdida de efectividad, ya que se trata de medicamentos indispensables. Los autores recomiendan instituir dos tipos de control de la calidad, uno para medicamentos de la red de distribución oficial y otro para los del mercado paralelo, puesto que las condiciones de almacenamiento respectivas son bastante diferentes. A la vez, ese control de calidad sería útil para detectar el ya emergente problema de los medicamentos falsificados y poder así prevenir graves consecuencias en la salud de la población. (Ballereau F, Prazuck T, Schrive I, Lafleuriel MT. Rozed D, Fisch A, et al. Stability of essential drugs in the field: results of a study conducted over a two-year period in Burkina Faso. Am J Trop Med Hyg 1997;57:31-36.) 